\title{
Version auteur
}

(Texte publié dans Histoire de l'art, 57 (2005) 3-13)

\section{Vingt ans de recherches sur la sculpture française des XVIe et XVIIe siècles.}

\author{
Marion Boudon-Machuel
}

$\mathrm{Au}$ milieu des années 1980, F. Souchal estimait que «le manque d'intérêt pour l'histoire de la sculpture se traduit d'abord par l'absence de mises au point des problèmes et des travaux $»^{1}$. Quelques vingt années plus $\operatorname{tard}^{2}$, un état des lieux est nécessaire pour comprendre où en est la recherche sur la sculpture française des XVIe et XVIIe siècles ${ }^{3}$ et quelles sont les perspectives qui s'offrent à elle.

Force est de constater que la sculpture occupe depuis longtemps une place réduite dans les travaux des historiens de l'art français. La recension des articles et ouvrages parus dans les vingt dernières années, confirme cette tendance, d'autant plus sensible que ceux sur la peinture et l'architecture ont connu, à l'inverse, un développement croissant ${ }^{4}$. Le plus inquiétant est que le petit nombre des publications semble être le reflet d'un désintérêt plus général pour la matière, qui touche tant le monde de la recherche que celui du marché de l'art, et qui conduit à distinguer la France de ses voisins européens et des Etats-Unis.

La responsabilité des chercheurs, surtout des universitaires, est de taille : matière peu, si ce n'est pas, enseignée, la sculpture est peu étudiée. C'est en effet essentiellement au sein des

\footnotetext{
* Cet article est en partie le fruit d'une enquête menée auprès de chercheurs spécialistes ou non de sculpture (de l'université, des musées, de l'inventaire...); je remercie tous ceux qui ont pris le temps de me répondre. Le recensement ainsi réalisé a, en particulier, bénéficié de l'œil le plus critique et averti de la sculpture française des XVIe et XVIIe siècles, celui de G. Bresc-Bautier.

Abréviations utilisées : Bulletin de la Société de l'Histoire de l'Art français(BSHAF); Gazette des Beaux-Arts (GBA), Nouvelles archives de l'art français (NAAF).

${ }^{1}$ F. Souchal, « La sculpture française des XVIIe et XVIIIe siècles. Etat des travaux », Formes, n ${ }^{\circ}$ 19, pp. 21-26.

${ }^{2}$ Aucune synthèse des recherches sur la sculpture française des XVIe et XVIIe siècles n'a été établie depuis vingt ans. Les dernières références, qui datent du milieu des années 1980 et couvrent partiellement la période, sont l'article de F. Souchal mentionné à la note précédente, et celui de G. Bresc-Bautier, «La statuaire sous Louis XIV », Revue de l'art, ${ }^{\circ}$ 69, 1985, pp. 69-72.

${ }^{3}$ Pour cette recension, nous avons volontairement choisi une période chronologique large, les XVIe et XVIIe siècles, en débordant sur la fin du XVe siècle et sur le début du XVIIIe siècle, étant entendu que la Renaissance ne commence pas strictement en 1494 ou en 1540, et que le XVIIe siècle ne se clôt pas l'année de la mort de Louis XIV, en 1715. Plus qu'une histoire de la sculpture, qui est encore à écrire, nous pensons que c'est le sujet qui peut permettre de fixer des limites chronologiques cohérentes, même s'il conduit à nier les périodisations universitaires.

${ }^{4}$ Pour l'architecture, voir Cl. Mignot, «Vingt ans de recherches sur l'architecture française (1540-1708)», Histoire de l'art, $\mathrm{n}^{\circ}$ 54, juin 2004, pp. 3-12.
} 
musées ou à l'étranger que les principaux travaux sont produits. Le nom de F. Souchal mis à part (mais ses élèves n'ont pas intégré l'université française), les auteurs des études sur la sculpture française des XVIe et XVIIe siècles sont majoritairement des conservateurs de musées (les publications de G. Bresc-Bautier dominent les deux siècles par la qualité, le nombre et la diversité des sujets abordés), des chercheurs indépendants (comme Fr. de La Moureyre) et des étrangers, notamment américains et allemands.

On comprend alors d'autant mieux l'apparente frilosité des éditeurs, voire des revues. Il n'existe toujours pas de synthèse spécifique à la production sculptée en France pour chacun de ces deux siècles. On doit donc se contenter des chapitres qui lui sont consacrés dans des ouvrages généraux sur la sculpture, tous siècles et tous pays confondus, ou sur l'art français d'une période donnée 5 . L'élan enregistré naguère - dans les années 1967-1968, les éditions Picard publiaient de grandes monographies, celle consacrée à Philippe de Buyster par P. Chaleix, à Matthieu Jacquet par E. J. Ciprut, et à Pierre de Franqueville par R. de Franqueville, et la maison De Boccard, la thèse de F. Souchal sur les Slodtz -, s'est beaucoup ralenti par la suite. Arthena ne compte à son actif que deux monographies de sculpteurs pour le XVIIe siècle dont une seule, la plus ancienne, est celle d'un artiste français ${ }^{6}$. Parmi les revues, certaines ont accueilli généreusement des articles sur la sculpture. À cet égard, la disparition de la Gazette des Beaux-Arts, qui, grâce à F. Souchal et à F. de La Moureyre, en était une des premières tribunes, est ici particulièrement ressenti. Le principal support national reste aujourd'hui le Bulletin de la Société de l'Histoire de l'Art français, devant la Revue de l'art. En revanche, les revues régionales (Pays Lorrain, La Vie en Champagne, 303 : Arts, Recherches et Création) s'efforcent de faire connaître la richesse de certaines régions, et les revues étrangères (The Art Bulletin, The Burlington magazine dans son numéro du mois de décembre, et le jeune Sculpture Journal) publient régulièrement des articles sur la sculpture française.

\footnotetext{
5 La sculpture de la Renaissance au XXe siècle, Milan, 1996, rééd. Cologne, Londres, Paris, 1999; The Encyclopedia of Sculpture, New-York, 2004. Pour la Renaissance, on se reportera à la réflexion riche et problématisée de H. Zerner dans L'art de la Renaissance en France. L'invention du classicisme, Paris, 1996. Il existe heureusement un ouvrage sur la technique de la sculpture, M.-Th. Baudry, Sculpture. Méthode et vocabulaire, Paris, 1978, rééd. 2000.

${ }^{6}$ M. Beaulieu, Robert Le Lorrain (1666-1743), Neuilly-sur-Seine, 1982. L'autre monographie pour le XVIIe siècle est celle sur François du Quesnoy (M. Boudon-Machuel, 2005) qui porte à trois, avec celle sur Laurent Delvaux pour le XVIIIe siècle (A. Jacobs, 1999) le nombre des monographies de sculpteurs de la période moderne publiées par Arthena. Ces ouvrages laissent augurer une orientation plus déterminée de cet éditeur à l'égard de la sculpture. Il faut signaler encore l'effort des éditions Macula pour traduire et publier des ouvrages fondamentaux sur la sculpture (E. Panofsky, La sculpture funéraire de l'ancienne Egypte au Bernin, Paris, 1995, R. Wittkower, Qu'est-ce que la sculpture? Principes et procédures, de l'Antiquité au XXe siècle, Paris, 1995 et J. von Schlosser, Histoire du portrait de cire, Paris, 1997).
} 
Le constat pourrait être accablant. Nous ne le pensons pas. Une nouvelle tendance se distingue. Depuis une dizaine d'années, de jeunes universitaires, certes peu nombreux, se sont tournés vers la sculpture. Les thèses, tant de doctorat que d'habilitation, soutenues ces cinq dernières années, laissent espérer un nouveau souffle qui devrait profiter du dynamisme des musées. Reste à l'université à savoir accueillir tant des enseignants qu'un enseignement plus spécialisé de sculpture.

\section{Connaître le sculpteur sous toutes ses faces}

Pour les deux siècles concernés, on connaît encore mal les acteurs principaux. Le XVIe siècle et la première moitié du XVIIe siècle font figures de parents pauvres : pour les cent cinquante ans, si riches en artistes et en œuvres, on ne compte que deux monographies (Dominique Florentin et Nicolas Blasset), deux expositions monographiques (Ligier Richier, Jacques Sarazin) et un colloque (Germain Pilon) ${ }^{7}$. Les ouvrages de référence sont souvent fort anciens (sur Michel Colombe celui de Vitry date de 1901, et celui de Pradel, de $1953^{8}$, sur Jean Goujon, la monographie de Du Colombier a été publiée en 1949), et des monographies utiles, comme celles sur Gérard van Opstal et Gilles Guérin, sont restées à l'état manuscrit ${ }^{9}$. Citons néanmoins, même s'ils ne concernent pas à proprement parler la sculpture française, les travaux consacrés à des sculpteurs français qui ont fait carrière hors du royaume en Italie, comme Nicolas Cordier, ou en Espagne, comme Etienne Jamet ${ }^{10}$. Ces exemples sont rares ${ }^{11}$;

\footnotetext{
${ }^{7}$ Monographies: I. B. Wardropper, The sculpture and prints of Domenico del Barbiere, Ph. D., New-York University, 1985, University Microfilms International, Ann Arbor (Mich.), 1994 ; Chr. Debrie, Nicolas Blasset (1600-1659), Paris, 1984. Catalogues d'expositions : Ligier Richier et la sculpture en Lorraine au XVIe siècle, Bar-Le-Duc, 1985 (citons également pour Richier l'ouvrage de B. Noël et P. Choné, Ligier Richier, Thionville, 2000 dans lequel sont étudiées les principales œuvres du sculpteur) ; Jacques Sarazin : sculpteur du Roi, 1592 1660, Musée du Noyonnais, Paris, Noyon, 1992. Colloques: Germain Pilon et les sculpteurs français de la Renaissance, dir. G. Bresc-Bautier, Paris, Musée du Louvre, 26-27 octobre 1990, Paris 1993. Pour le colloque consacré à Michel Colombe, voir note suivante.

${ }^{8}$ Le récent colloque sur Michel Colombe laisse de nombreuses questions en suspens, comme le souligne J.-R. Gaborit dans sa présentation (Michel Colombe et son temps, actes du $124^{e}$ Congrès des sociétés historiques et scientifiques, section histoire de l'art et archéologie, Nantes, 19-26 avril 1999, Paris, 2001).

${ }^{9}$ G. Bue-Akar, Van Opstal, mémoire de l'école du Louvre, 1975 ; M. Th. Forest, Gilles Guérin, Paris 1611/12Paris 1676, maîtrise, Paris IV, 1975. Les articles publiés par ces auteurs ne viennent pas combler l'absence d'un catalogue raisonné.

${ }^{10}$ S. Pressouyre, Nicolas Cordier : recherches sur la sculpture à Rome autour de 1600, Rome, 1984 et A. Turcat, Étienne Jamet alias Esteban Jamete, sculpteur français de la Renaissance en Espagne condamné par l'Inquisition, Paris, 1994.

${ }^{11}$ On peut ajouter néanmoins l'exemple d'Hubert Le Sueur dont la carrière en Angleterre et en France a pu être reconstituée (Ch. Avery, « Hubert Le Sueur, the 'Unworthy Praxiteles' of King Charles I », The Walpole Society, XLVIII, 1982, pp. 132-209, republié dans Studies in European Sculpture II, Londres, 1988, pp. 145-235 et complété par G. Bresc-Bautier, "L'activité parisienne d'Hubert Le Sueur sculpteur du roi (connu de 1656 à 1658) », BSHAF, 1985, (1986), pp. 35-54).
} 
le plus souvent les sculpteurs français à l'étranger n'ont pas réellement percé, ou bien leur séjour ne couvre qu'une partie de leur carrière, en amont ; les documents et les œuvres sont alors difficiles à trouver, voire inexistants ${ }^{12}$ et n'incitent pas à la recherche. Des travaux récents ont, en revanche, éclairé l'activité de sculpteurs étrangers en France, en particulier italiens comme Girolamo della Robbia, Rustici et Cellini ${ }^{13}$.

Il en va autrement pour le règne de Louis XIV. Dès la fin des années 1970 et au cours des années 1980, il a bénéficié des trois précieux volumes des French Sculptors : une notice est consacrée à chacun des sculpteurs du roi, nourrie de pièces d'archives et d'illustrations les plus exhaustives possible ${ }^{14}$. Dans les mêmes années, sont parues des monographies sur les frères Coustou, Robert Le Lorrain et les frères Marsy ${ }^{15}$, parfois sous la forme d'expositions à Marseille et à Gêne pour Pierre Puget, au Puy-en-Velay pour Pierre Vaneau ${ }^{16}$. Et pourtant, ici encore les recherches se sont ralenties. Pour les deux grands acteurs du règne, François Girardon et Antoine Coysevox, les monographies de base restent celles de Francastel (1928) pour le premier - on bénéficie heureusement d'une étude plus récente sur une partie de la

\footnotetext{
${ }^{12}$ Ainsi pour les sculpteurs qui se sont rendus à Rome au XVIIe siècle, les documents d'archives trouvés par J. Bousquet offrent une première piste qu'il est souvent difficile de poursuivre (le titre de son ouvrage Recherches sur le séjour des peintres français à Rome au XVIIe siècle, Montpellier, 1980, est trompeur, il concerne aussi les sculpteurs) ; lorsque l'on peut aller plus loin, ce sont les œuvres qui font défaut (voir par exemple M. Boudon-Machuel, «Un autre Claude Lorrain à Rome : le sculpteur Claude Pernet», Bulletin de l'Association des Historiens de l'Art Italien, $\mathrm{n}^{\circ} 10,2004$, pp. 23-41). Signalons encore un mémoire de maîtrise sur La chair à prêcher de l'église Santa Cruz de Coimbra et les sculpteurs français de la Renaissance au Portugal (P. Alloin, Aix-Marseille I, 1999).

13 Th. Crépin Leblond, «Le retable de la chapelle de Cognac et l'influence de Girolamo della Robbia en France », BSHAF, 1996, (1997), pp. 9-20 ; B. Jestaz, «Benvenuto Cellini et la cour de France (1540-1545)», Art et artistes en France de la Renaissance à la Révolution, Paris, 2003, pp. 71-132. La même année sont parus deux articles sur la participation de Cellini à la Porte Dorée de Fontainebleau : J. R. Bliss, « Benvenuto Cellini's Satyrs for the Porte Dorée at Fontainebleau» dans Large Bronzes in the Renaissance, éd. P. Motture, Washington, 2003, pp. 72-93 et J. Mardsen et J. Bassett, «Cellini's other Satyr for the Porte Dorée », The Burlington Magazine, CXLV, n¹205, août 2003, pp. 552-563. L. Châtelet-Lange, «Benvenuto Cellini und « der gute König Franz » : der Künstler als Günstling », dans Der Fall des Günstlings : Hofparteien in Europa vom 13 bis zum 17 Jahrhundert : 8. Symposium der Residenzen-Kommission der Akademie der Wissenschaften zu Göttingen, Ostfildern, 2004, pp. 489-494. Pour Rustici, on attend la publication de la thèse d'habilitation de Ph. Sénéchal (Giovanni Francesco Rustici. Un sculpteur florentin de la Renaissance entre Florence et Paris, Paris IV, 2003) et de la thèse de doctorat de M. K. Minning (Giovan Francesco Rustici (1475-1554). Untersuchungen zu Leben und Werk des Florentiner Bildhauers. Münster, Westfälische Wilhelms-Universität, 2004). Hormis ces grandes figures d'artistes étrangers, mentionnons encore des sculpteurs de moindre envergure comme Jan Crocq pour le début de la période (P. Simonin, « Euvres de 'Jan Crocq, sculpteur Néerlandais en Lorraine' », Le Pays lorrain, 100, 2003, pp. 194-196).

${ }^{14}$ French Sculptors of the 17 th and 18th centuries: The reign of Louis XIV (sous la direction de F. Souchal avec la collaboration de F. de La Moureyre et H. Dumuis), Oxford, 1977 (vol. I, A à F), 1981 (vol. II, G à L), 1987 (vol. III, M à Z), et une mise à jour en 1993 (vol. IV, A à Z).

${ }^{15}$ F. Souchal, avec la collaboration de Fr. de La Moureyre, Les Frères Coustou, Paris, 1980 ; M. Beaulieu, Robert Le Lorrain (1666-1743), Neuilly-sur-Seine, 1982 et Th. Hedin, The Sculpture of Gaspard and Balthazar de Marsy. Art and Patronage in the early Reign of Louis XIV, with a catalogue raisonné, Columbia, 1983.

${ }^{16}$ La Sculpture française au XVIIe siècle. Pierre Vaneau, Le Puy-en-Velay, 1980. Pierre Puget, peintre, sculpteur, architecte 1620-1694, Marseille-Paris, 1994 et la version italienne qui comporte quelques variantes, Pierre Puget (Marsiglia 1620-1694), un artista francese e la scultura barocca a Genova, Milan, 1995.
} 
carrière $^{17}$ - et de Keller-Dorian (1920) pour le second. On n'oserait se plaindre encore des ouvrages fondamentaux parus en allemand et jamais traduits comme la monographie de $\mathrm{K}$. Herding sur Puget (1970) ou celle de L. Seelig sur Desjardin (1980), ni des travaux restés à l'état manuscrit ${ }^{18}$.

Doit-on penser que la monographie d'artiste n'est plus un genre adapté ? Cet exercice souvent critiqué, considéré par certains comme désuet et réduit à une approche parfois qualifiée, sans trop de souci épistémologique, de «positiviste », semble pourtant loin d'être obsolète en la matière. Comment établir des synthèses, comment développer des analyses transversales fondées alors qu'il n'existe pas même de monographies récentes ou complètes sur des acteurs majeurs de la période ? On a ainsi peut-être trop vite pensé, allant d'ailleurs à l'encontre de l'avis même de ses auteurs, que le dictionnaire des French sculptors rendait superfétatoire toute étude approfondie sur un des sculpteurs du temps de Louis XIV. Sans doute, la richesse de l'ouvrage a pu, un temps, bloquer les initiatives. Ce répertoire de documents et d'œuvres doit néanmoins être considéré pour ce qu'il est : un outil essentiel pour de nouvelles recherches tant monographiques que thématiques; des travaux universitaires récents le prouvent ${ }^{19}$. Pour l'ensemble de la période, un autre dictionnaire est devenu indispensable. L'Allgemeines Künstlerlexikon, plus complet que son aîné le Thieme und Becker $^{20}$, publie désormais tant des œuvres que des pièces d'archives inédites ${ }^{21}$, et la version sur CDRom constitue aujourd'hui l'outil indispensable pour interroger cette mine d'informations. Surtout, les publications récentes en témoignent, le genre monographique n'est évidemment pas immuable. L'essai critique peut y acquérir une plus grande autonomie. Loin de paraphraser le catalogue, il explique l'artiste et son œuvre dans le contexte plus large auquel ils appartiennent comme l'a brillamment démontré S. Pressouyre avec son Nicolas

\footnotetext{
${ }^{17}$ D. Walker, The early career of François Girardon, 1626-1686 : the history of a sculptor to Louis XIV during the superintendance of Jean-Baptiste Colbert, Ph. D., New-York University, 1982.

${ }^{18}$ Les ouvrages de S. Hoog, Etienne Le Hongre, mémoire de l'école du Louvre, 1960 et de G. Walton, The Sculptures of Pierre Puget [1967] n'ont jamais été publiés.

${ }^{19}$ F. Sartre, Marc Arcis, un Toulousain sculpteur du roi (1652-1739) (Toulouse II, 1999).

${ }^{20} \mathrm{G}$. Fries nous a fourni des statistiques éloquentes : sur les 43 premiers volumes, on relève presque 1400 noms de sculpteurs français ou actifs en France pour la période retenue ici (parmi lesquels moins de 990 figuraient déjà dans le Thieme und Becker, et seulement 439 dans le Lami).

${ }^{21}$ Les notices enregistrées dans la version sur CDRom, qui comprennent des mentions de pièces d'archives, s'élèvent à 529. Citons deux cas notables d'inédits publiés dans l'AKL sur lesquels G. Fries a très aimablement attiré notre attention. Le buste en marbre de Martin Fréminet du Louvre, en quête d'auteur depuis deux siècles, peut être désormais donné, à l'appui de pièces d'archives, à Barthélemy Tremblay (voir vol. 44). Plus étonnant, le repérage de la rédaction de l'AKL, a conduit à voir non pas deux, mais une personne, en Imbert Boachon, architecte et sculpteur dont le style est, il est vrai, hétérogène, et, au-delà, à reconsidérer un éventuel séjour de l'artiste en Val-de-Loire, ou même en Italie du Nord pour expliquer cette rupture stylistique (E. Mognetti, notice sur Boachon dans le vol. XI et $i d$., «Italianisme(s) dans la sculpture avignonnaise de Francesco Laurana à Imbert Boachon », dans Du gothique à la Renaissance : architecture et décor en France (1470-1550), actes du colloque de Viviers, 20-23 septembre 2001, dir. Yves Esquieu, Aix-en-Provence, 2003, pp. 17-32).
} 
Cordier. Il faut donc souhaiter que des travaux universitaires sur des sculpteurs du XVIe siècle, comme Germain Pilon, Ponce Jacquio et Jean Guiramand, ou du XVIIe siècle, comme Barthélemy Prieur, les Bourdin, Pierre Affre et Marc Arcis, pour ne citer que les principaux ${ }^{22}$, donnent lieu à des publications ${ }^{23}$.

Cependant, pour l'étude des sculpteurs français des XVIe et XVIIe siècles, il faut reconnaître que l'approche monographique touche à ses limites. Contrairement à la peinture et à l'architecture, on compte moins d'artistes de renom ; surtout, la production est beaucoup moins abondante, les œuvres sont plus petits et, qui plus est, moins bien conservés. D'autres formes d'études de type monographique, non plus seulement sur le sculpteur et son œuvre mais sur une communauté d'artistes, se sont ainsi développées, offrant un regard élargi. Les expositions sur Sarazin et surtout sur Puget ont réservé une place distincte aux sculpteurs de «l'atelier»; récemment, celles sur les terres cuites du Maine ou la sculpture à Toulouse au XVIIe siècle, fruits de plusieurs années de recherches à plusieurs mains, ont conduit à distinguer des personnalités artistiques au sein de communautés de sculpteurs ${ }^{24}$. Des travaux universitaires relèvent pour partie de cette approche, livrant un panorama plus vaste encore

\footnotetext{
${ }^{22}$ Maîtrises inscrites ou soutenues: Chr. Benoist, Recherches sur les sculpteurs Michel Bourdin, père et fils (Paris IV, 1987) ; S. Alibert, François Marchand et la sculpture à Chartres et à Orléans (Paris IV, 1995) ; Y. Helfer, Ponce Jacquio : l'inaccessible étoile? (Paris IV, 2001). Citons encore E. Beauville, L'atelier des Tournier, sculpteurs sur bois au XVIIe siècle dans le Gourdonnais (Bordeaux III, 1988); St. Martinati, Le sculpteur Jacques Bousseau (1681-1740) (Paris I, 1993); A.-L. Quitte, Jean II Girouard, sculpteur poitevin. XVII-XVIIIe siècles (Poitiers, 1997) ; S. Chabre, Christophe Veyrier, un sculpteur dans l'ombre de Puget, 16371689 (Aix-Marseille I, 2001); D.E.A.: N. Tailliez, Les euvres roussillonnaises de Jean-Jacques Mélair, sculpteur carcassonnais du XVIIe siècle (Montpellier III, 1998), de C. Voiriot, Jean Voiriot (1672-1740), sculpteur des bâtiments du roi (Paris IV, 2000), A. Dulac, Le sculpteur du roi Jean-Jacques Clérion (1637-1714) (Aix-Marseille, 2001), A. Adamczak, Jean-Baptiste Théodon, sculpteur (1645-1713) (Paris IV, 2001). Thèses: C. Bourdieu, Pierre Affre et la sculpture toulousaine de 1630 à 1670 (Toulouse II, 1994) publiée partiellement, voir C. Bourdieu, «Pierre Affre et l'art du retable à Toulouse au XVIIe siècle», dans Toulouse et le midi toulousain entre ciel et terre du Moyen Age à nos jours, actes du 47 congrès de Toulouse 11-13 juin 1993 et id., «Pierre Affre » et «l'atelier de Pierre Affre dans le catalogue de l'exposition L'Age d'or de la sculpture...,, Chr. Galissot, Jean Guiramand et la sculpture en Basse-Provence dans la première moitié du XVIe siècle (AixMarseille, 2001) ; F. Sartre, Marc Arcis..., op. cit. Les thèses de chercheurs étrangers comme celles de Mary L. Levkoff sur Germain Pilon, ou de R. Seelig-Teuwen sur Prieur sont également en attente d'être publiées.

${ }^{23}$ Certains travaux de maîtrise ont été publiés partiellement ou en totalité : M. Boudon, «L'imagier Jacques Juliot et le retable de l'abbaye de Larrivour », BSHAF, 1997, (1998), pp. 9-30 ; O. Geneste, Les Duhamel, sculpteurs à Tulle aux XVIIe et XVIIIe siècles, Limoges, 2002.

${ }^{24}$ P. Julien a publié un article monographique sur Gervais Drouet ( $«$ Un Disciple du Bernin : Gervais Drouet, sculpteur toulousain », BSHAF, 1994, (1995), pp. 67-98), et C. Bourdieu un article sur Pierre Affre (op. cit note 22.) avant de participer à l'exposition L'Age d'or de la sculpture..., op. cit. De même, les articles sur Hoyau et Delabarre ont permis de dégager l'œuvre de ces sculpteurs et de préparer l'exposition Terre et ciel. La sculpture en terre cuite du Maine, XVIe et XVIIe siècles, ouvrage collectif publié à l'occasion de l'exposition de l'abbaye de l'Epau dans les Cahiers du patrimoine, juin, 2003. Voir G. Bresc-Bautier, et J. Perrin, avec la collaboration de F. Le Bœuf, «Charles Hoyau, sculpteur du Mans », BSHAF, 1991, (1992), pp. 37-64 ; et dans le même numéro, Fr. de La Moureyre avec la participation de J. Perrin et G. Bresc-Bautier, «Mieux vaut un vrai Delabarre qu'un faux Sarazin : le tombeau de Donadieu de Puycharic et le sculpteur Gervais Delabarre », pp. 6590 (contrairement à ce que laisse à penser le titre, l'article ne concerne pas que le tombeau de Donadieu de Puycharic, mais présente également, par ordre chronologique, les éléments de la biographie et les œuvres de Delabarre, ainsi que des sculptures qui lui sont attribuées sans datation).
} 
pour les sculpteurs et la production sculptée d'une période en un lieu donné ${ }^{25}$. De même, c'est peut-être moins en partant du sculpteur, que du système de production ou d'un type d'œuvres dans lequel il s'est illustré - Girardon et la notion d'atelier, Coysevox et le portrait sculpté, pour citer quelques exemples - que de grandes figures de la période pourront être réétudiées.

Parallèlement, on a réussi à mieux cerner le sculpteur au sein de sa profession, en exploitant notamment les sources que les chercheurs, dans les années 1960-1970, avaient récoltées avec un soin particulier ${ }^{26}$. Les documents d'archives qui concernent les chantiers royaux, relativement abondants et regroupés, ont permis de suivre le travail des sculpteurs pour les règnes de François Ier et d'Henri $\mathrm{II}^{27}$. Le statut et le mode de travail de ces artistes d'une région ou d'une ville, suivis sur une longue période ${ }^{28}$, révèlent parfois des limites floues avec des professions voisines (menuisiers, fondeurs) ${ }^{29}$. Pour le XVIIe siècle, le statut des sculpteurs ainsi que les relations contractuelles avec d'autres artistes ont été analysés à la lumière de nouvelles pièces d'archives ${ }^{30}$. Enfin, à partir de vastes dépouillements de fonds

\footnotetext{
${ }^{25}$ Voir la partie consacrée aux sculpteurs dans C. Chédeau, Les Arts à Dijon au XVIe siècle : les débuts de la Renaissance 1494-1551, Aix-en-Provence, 1999, pp. 130-178. Pour le XVIIe siècle, signalons la thèse de D. Fernandez, Architecture, sculpture et décoration à Dijon au temps de Jean et Guillaume Dubois (1625-1738) (Paris IV).

${ }^{26}$ Rappelons que pour les deux siècles étudiés, les principales sources ont été publiées dans les années 19601980 : E.-J. Ciprut, Documents inédits (histoire, littérature, arts) XVI-XVIIIe siècles, Paris, 1965 ; M.-A. Fleury, Documents du Minutier central concernant les peintres, les sculpteurs et les graveurs au XVIIe siècle (16001650), Paris, t. I, 1969 ; C. Grodecki, Documents du minutier central des notaires de Paris. Histoire de l'art au XVIe siècle (1540-1600) II. - Sculpture - Peinture - Broderie - Émail et Faïence - Orfèvrerie - Armures, Paris, 1986. Enfin, parmi les nombreuses sources publiées par R. Blant, on peut citer ses « Sculpteurs du temps d'Henri IV d'après les documents du minutier central à Paris », dans les Actes du $96^{e}$ Congrès national des sociétés savantes. Archéologie et histoire de l'art, Toulouse, 1971, t. 1, pp. 399-414, et « Sculpteurs épitaphiers des $16^{\mathrm{e}}$ et $17^{\mathrm{e}}$ siècles d'après les actes inédits du minutier central à Paris », dans les Actes du $99^{e}$ congrès national des sociétés savantes. Archéologie et histoire de l'art, Besançon, 1974, pp. 321-338.

${ }^{27}$ G. Bresc, «Les sculpteurs de Primatice », dans Primatice, maître de Fontainebleau, Paris, musée du Louvre, 2004-2005, pp. 31-37, et id. "Les sculpteurs des Bâtiments du Roi sous Henri II», actes du colloque international Henri II et les arts, École du Louvre et musée national de la Renaissance, Écouen, 25-27 septembre 1997, dir. d'H. Oursel et J. Fritsch, Paris, 2003, pp. 117-133. On trouvera également des références utiles sur la corporation des peintres et des sculpteurs à Paris sous François Ier dans G.-M. Leproux, La peinture à Paris sous le règne de François Ier, Paris, 2001.

${ }^{28} \mathrm{C}$. Chédeau « Réflexions sur l'organisation des ateliers de sculpteurs en Bourgogne et en France aux XVe et XVIe siècle : les modèles d'atelier », dans Pierre, lumière, couleur, Etudes d'histoire de l'art du Moyen Âge en l'honneur d'Anne Prache, Cultures et civilisations médiévales, 20, 1999, pp. 487-499, F. Le Boeuf, «Les Sculpteurs terracottistes du Maine », 303 : Arts, Recherches et Création, 2003, 77, pp. 4-11, Terre et ciel... op. cit.

${ }^{29}$ G. Bresc, « Problèmes du bronze français : fondeurs et sculpteurs à Paris (1600-1660), NAAF, t. XXX, 1989, pp. 11-50, id., «Parisian Caster in the Sixteenth Century », dans Large Bronzes... op. cit. pp. 95-113 et id., « Le petit bronze », dans Un temps d'exubérance. Les arts décoratifs sous Louis XIII et Anne d'Autriche, Paris, Galerie Nationale du Grand Palais, 9 avril-8 juillet 2002, Paris, 2002, pp. 422-423 ; Agnès Magnien, « Les bronzes 'Keller' », BSHAF, 1996 (1997), pp. 37-63 ; dans C. Chédeau, Les Arts à Dijon au XVIe siècle ... op. cit, pp. 130-134 ; G. Bresc, et M. Constans, «Toussaint Chenu, un sculpteur parisien 'si connu dans la ville' », dans Etudes d'histoire de l'art offertes à Jacques Thirion, 2001, pp. 197-212.

${ }^{30}$ G.-M. Leproux, «Les corporations des peintres et sculpteurs à Paris dans les premières années du XVIIe siècle », XVIIe siècle, n²10, pp. 649-668. Pour les relations contractuelles entre sculpteurs, on pourra consulter
} 
d'archives, on commence à exploiter de façon nouvelle les relations des sculpteurs avec d'autres agents économiques liés à leur activité, comme par exemple avec les marbriers ${ }^{31}$.

\section{Les sculptures et la Sculpture}

Le recensement des sources est plus avancé que celui des œuvres. Quand ils ne manquent pas, les inventaires de la sculpture sont insuffisants. L'utile base Palissy, effort commun des Monuments Historiques et de l'Inventaire, qui devrait contenir à terme le corpus complet des sculptures conservées en France, couvre inégalement, et selon une progression aléatoire l'ensemble du territoire. Même des régions riches de travaux anciens, comme la Champagne ou la Lorraine, constituent des mines trop peu exploitées ${ }^{32}$. Pourtant l'étude des œuvres produites dans une région est certainement l'une des voies à privilégier pour aborder la sculpture, en particulier à la Renaissance ${ }^{33}$.

Plus fréquemment, c'est autour d'un thème que les sculptures d'une région ont été rassemblées et publiées, sans réel souci d'exhaustivité ${ }^{34}$. Par nature, le mobilier religieux,

Th. Hedin, «Exemple d'une collaboration d'artistes : the Partnership pf Gaspard Marsy and Anselme Flamen, 1679-1681 », GBA, 1981, 2, pp. 104-114.

${ }^{31}$ On a beaucoup travaillé ces dernières années sur les marbres royaux. Pour la période retenue ici, on consultera G. Bresc, «Le Marbre du roi : L'Approvisionnement en marbre des bâtiments du roi, 1660-1715 », actes du colloque The Art and Architecture of Versailles, 1985, dans Eighteenth Century Life, vol. 17, 2, mai 1993, pp. 37-54. Trois recherches de fond sur les marbres sont en cours de publication : 1'ouvrage de G. Bresc, La guerre du marbre. Le commerce du marbre de Carrare en France sous Louis XIV, la thèse de Sophie Mouquin, Les marbriers des Bâtiments du Roi, 1661-1745, (Paris IV, 2003) et les actes du colloque Marbres de Rois, Versailles, 22-24 mai 2003. Sur l'intervention des sculpteurs dans l'approvisionnement en marbre statuaire, on pourra se reporter plus spécifiquement à l'intervention dans ce colloque de M. Boudon-Machuel et F. Sartre «Le combat n'est point douteux, voici un bloc qui s'offre à vous servir. Le sculpteur et l'approvisionnement en marbre statuaire ».

${ }^{32}$ Il est remarquable que l'ouvrage de référence pour l'étude de la sculpture champenoise du XVIe siècle soit toujours celui de R. Koechlin et J.-J. Marquet de Vasselot, La sculpture à Troyes et dans la Champagne méridionale au XVIe siècle, Paris, 1900 et, pour la sculpture en Lorraine, celui de H. van Hees, Die lothringische Skulptur des 16. Jahrhunderts, Sarrebruck, 1973, jamais traduit en français. Les travaux de A.-F. Arnhold (Le jubé de la Madeleine et la sculpture à Troyes autour de 1500, D.E.A., Paris IV, 1991 et Die Skulptur in Troyes und der südlichen Champagne zwischen 1480 und 1540, thèse de doctorat, Fribourg, 1992) ne sont pas publiés. On peut saluer l'entreprise de recensement des œuvres, canton par canton, récemment entrepris sous la direction de P. Corbet et P. Sesmat: S. Derson, Corpus de la statuaire médiévale et Renaissance de Champagne méridionale, 1. Canton de Soulaines-Dhuys (Aube), Langres, 2003 et P. Corbet et M.-F. Jacops, Corpus de la statuaire médiévale et Renaissance de Champagne méridionale, 1I. Canton de Doulevant-le-Château (HauteMarne), Langres, 2004. Les ouvrages de J. Baudoin, souvent cités, sont avant tout utiles pour les photographies (J. Baudoin, La sculpture flamboyante : les grands imagiers d'Occident, Nonette, 1983 ; id., La sculpture flamboyante, Champagne-Lorraine, Nonette, 1991 ; id., La sculpture flamboyante en Normandie et en Ile-deFrance, Nonette, 1992).

${ }^{33}$ Des mémoires de l'Ecole du Louvre sur ce type de sujets ont produit d'excellents résultats comme celui de G. Benoit Ecolan, La Sculpture de la Renaissance dans le Vexin français (1999), qui est en cours de publication, ou celui d'O. Stublier, La sculpture religieuse dans l'ancien comté de Nice aux XVIIe et XVIIIe siècles (1988).

${ }^{34}$ Signalons les études proches de l'inventaire ou de la synthèse autour d'un thème iconographique, qu'elles soient régionales (c'est le cas des calvaires: Y.P. Castel, «Floraison des croix et calvaires dans le Léon sous l'influence de Monseigneur Roland Neufville (1562-1613) », Annales de Bretagne, 1983, 90, n² 2, pp. 311-320 ; $\mathrm{Ph}$. Bonnet, La légende de la vie autour de la mort: iconographie de la Mise au tombeau en Bretagne, s. 1., 2000), nationales (W. H. Forsyth, The Pietà in French late Gothic Sculpture : regional variations, New-York, 
stalles, chaires à prêcher, jubés, se prête plus facilement aux études typologiques ${ }^{35}$. Celles sur les retables surtout, depuis les ouvrages pionniers de V.-L. Tapié en Bretagne et M. Ménard pour la région mancelle ${ }^{36}$, ont suivi une progression régulière ; elles couvrent aujourd'hui plusieurs régions ${ }^{37}$ et pourraient être complétées notamment par des travaux universitaires ${ }^{38}$ pour peut-être, à terme, nourrir une analyse comparée à plus grande échelle.

Récemment, le matériau même des œuvres a été l'un des éléments retenus dans la définition d'un corpus. Ce fut le cas du bronze, associé à la notion de collection $^{39}$ pour l'exposition sur Les bronzes de la Couronne au Louvre en 1999, ou de la terre cuite, matériau privilégié de la production sculptée dans la région mancelle, présentée aux expositions Belles

1995 ; Fr. Baron, « La mise au tombeau dans la sculpture française de la Renaissance », Niccolo dell'Arca, actes du colloque 26-27 mai 1987, Bologne, 1989, pp. 213-219) ou à l'échelle de l'Europe (M. Martin, dans $L a$ Statuaire de la Mise au Tombeau du Christ des XVe et XVIe siècles en Europe occidentale, Paris, 1997, a recensé un nombre important de pièces françaises).

${ }^{35}$ F. Bonal, Les Stalles des églises de Champagne, Langres, Saints-Geosmes, 1997 ; P. Julien, D’Ors et de Prières. Arts et dévotions à Saint-Sernin de Toulouse, XVI-XVIIIe siècle, Aix-en-Provence, 2004 ; E. RoffidalMotte, Les stalles et la chaire de la basilique Saint-Maximin, Marseilles, 2000. Citont également les travaux universitaires Les chaires à prêcher à Toulouse (Fl. Dejoux, maîtrise, Toulouse II, 1994) ; Les jubés dans les Côtes d'Armor, XVe-XVIe siècles (M. Besnard, maîtrise, Rennes II, 1997), Les stalles en Ille-et-Vilaine, XIVeXIXe siècles (S. Leparoux, maîtrise, Rennes II, 1997), poursuivi en DEA pour l'ensemble de la Bretagne. Pour la sculpture religieuse dans son ensemble, j'ai été obligeamment autorisée à consulter avant sa parution l'Historiographie de l'histoire de l'art religieux en France à l'époque moderne et contemporaine. Bilan bibliographique (1975-2000) et perspectives, dirigée par J.-M. Leniaud et I. Saint-Martin, vaste recension critique qui englobe la sculpture.

${ }^{36}$ V.-L. Tapié, J.-P. Le Flem et A. Pardailhe-Galabrun, Retables baroques de Bretagne et spiritualité du XVIIe : étude sémiographique et religieuse, Paris, 1972 et M. Ménard, Une histoire des mentalités religieuses aux XVIIe et XVIIIe siècles. Mille retables de l'ancien diocèse du Mans, Paris, 1980.

${ }^{37}$ M. Brunet, «Un aspect du baroque provençal : les retables ruraux du Var», Provence historique, $\mathrm{n}^{\circ} 137$, juillet-septembre 1984, pp. 263-275 ; Y. Pelletier, Les retables bretons, Ouest-France, 1984 ; S. de Montessus de Ballore Lecointre, Retables et tabernacles des XVIIe et XVIIIe siècles dans les églises de la Creuse, Paris, 1988 ; D. Eraud, D. de Maynard, J. Perrin et J. Salbert, Retables de la Mayenne, Inventaire général des monuments et des richesses artistiques de la France, Images du Patrimoine, n², Nantes, 1990 ; P. Simonin, « Retables sculptés gothiques de la première Renaissance en Lorraine », Lotharingia, 1990, v. 2, pp. 199-270 ; L. Bouyssou, Retables de Haute-Auvergne XVIIe-XIXe siècles, Nonette, 1991 ; C. Bourdieu, «Pierre Affre et l'art du retable à Toulouse au XVIIe sièce », op. cit.; P. Boisnard, A. Flicoteaux, Ph. Hertel, JL Langrognet, G.-J. Michel, P. Mignery, La Haute-Sâne des retables : un langage artistique et symbolique des XVIIe et XVIIIe siècle au service du Sacré, Comité départemental du tourisme de la Haute-Saône, 2003 ; Regards sur les retables. Architectures ou théâtres d'images, dir. Chr. Langé et H. Palouzié, Paris, 2004. Un colloque intitulé L'Europe des retables, s'est tenu au Mans, à l'abbaye de l'Epau, les 13 octobre-16 octobre 2004.

${ }^{38}$ Les retables constituent un sujet privilégié pour des travaux de maîtrise si l'on en juge par le dépouillement des travaux d'étudiants depuis une dizaine d'années : Retables et tabernacles entre le XVIIe et le XIXe siècle dans le sud du Puy-de-Dôme (étude avec catalogue) (Chr. Vezon, Clermont-Ferrand II, 1993) ; Les retables baroques du Pays de Sault (C. Vacquié-Leménager, Toulouse, 1993) ; Etude historique et artistique des retables du XVIIe siècle en Franche-Comté (Br. Legrand, Besançon, 1996) ; Les retables du XVIIe et du XVIIIe siècle dans les églises de la Flandre maritime (V. de Lepeleire, Lille III, 1996) ; Les retables des XVIIe et XVIII siècles dans le nord-ouest du diocèse de Limoges (I. Tourneur, Limoges, 1996) ; Les retables des chapelles et du chœur de la basilique Saint-Maximin, $X V I^{e}-X V I I I^{e}$ siècles (M. Krohne, Aix-Marseille I). Signalons également, dans la continuité d'une maîtrise et d'un D.E.A., sur les retables de Tarentaise des XVIIe et XVIIIe siècles, la thèse en cours de É. Charabidze, intitulée Les retables des Alpes de Piémont-Savoie aux XVIIe et XVIIIe siècles (Paris XNanterre).

${ }^{39}$ Voir également S. Castelluccio, «Les collections de bronzes du Grand Dauphin », dans Curiosité. Etudes de l'art en l'honneur d'Antoine Schnapper, Paris, 1998, pp. 355-363. 
et inconnues au Louvre en 2002 et Terre et ciel à l'abbaye de l'Epau en $2003^{40}$. Les petits bronzes français en particulier fascinent d'autant plus qu'ils restent difficiles à saisir. Des catalogues de collections sont progressivement dressés ${ }^{41}$ et un groupe d'une trentaine de spécialistes internationaux s'est même constitué pour analyser ensemble les bronzes français des XVIIe et XVIIIe siècles conservés dans les grands musées européens, américains et russes ${ }^{42}$. Ces manifestations s'inscrivent dans une tendance plus générale qui vise à interroger l'œuvre par son matériau ${ }^{43}$, comme en témoigne notamment la publication de rapports de restauration - le cas le plus remarquable étant celui des stucs de la Galerie d'Apollon au Louvre -, qui rendent enfin accessible à un large public des éléments techniques essentiels à la compréhension des sculptures ${ }^{44}$.

Il serait impossible et peu pertinent de présenter ici les articles, nombreux, qui traitent d'une ou de quelques sculptures, généralement inédites; il est en revanche intéressant de souligner combien l'analyse des œuvres par leur sujet a conduit à approfondir deux thèmes féconds pour la statuaire des XVIe et XVIIe siècles : la symbolique du pouvoir et la sculpture funéraire. La sculpture est l'un des supports privilégiés de l'image du pouvoir. Les règnes de souverains soucieux d'asseoir leur légitimité, comme Henri IV, ou d'imposer leur pouvoir en chaque place du royaume, comme Louis XIV, ont donné lieu à une production généreuse d'effigies du roi, du buste à la statue pédestre ou équestre ${ }^{45}$. Moins immédiat, le reflet du

\footnotetext{
${ }^{40}$ op. cit.

${ }^{41}$ Voir notamment J. Marsden, «A checklist of French bronzes in the Royal Collection », Apollo, 156, 2002, $\mathrm{n}^{\circ}$ 486, pp. 47-49 et R. Wenley, French bronzes in the Wallace Collection, Londres 2003.

${ }^{42}$ The French bronze study group a été constitué à l'initiative de J. Marsden et R. Wenley.

${ }^{43}$ Ces dernières années, des expositions importantes ont centré une partie de leur propos sur le matériau (voir ici l'article de G. Bresc-Bautier). Pour le bronze en particulier, il faut mentionner l'important article d'Agnès Magnien (op. cit. ici note XX) consécutif à sa thèse de l'Ecole des Chartes.

${ }^{44}$ C'est le cas de la collection «Patrimoine restauré » des éditions du Patrimoine qui présente au public des restaurations d'œuvres importantes comme cela a été le cas pour le Sépulcre de Saint-Mihiel ou le Transi de Barle-Duc de Ligier Richier ( $n^{\circ} 3$ et 4 de septembre 2004). Les restaurations des stucs de la galerie d'Apollon font l'objet d'un long développement dans La galerie d'Apollon au palais du Louvre, dir. G. Bresc-Bautier, Paris, 2004 (G. Bresc «Les stucs de la galerie d'Apollon. Quatre sculpteurs, quatre tempéraments », pp. 99- 108; T. Borel, A. Bouquillon, F. Cerri, C. Chevillot, G. De Monte, C. Di Matteo, S. Pagès-Camagna et Daniel Vigears, " Le décor sculpté et doré au XVIIe siècle ", pp. 109-118 et «Duban et la restauration des sculptures, 1849$1851 »$, pp. 170-175; G. Bresc Bautier, C. Chevillot, G. De Monte, C. Di Matteo, «La restauration des sculptures », pp. 286-292).

45 A. Lefébure, «L'atelier de Barthélemy Prieur et l'imagerie royale sous le règne de Henri IV », dans L'avènement de Henri IV. Quatrième centenaire. Les arts au temps de Henri IV, Fontainebleau, 1992 et R. Seelig-Teuwen, «Barthélémy Prieur, portraitiste d'Henri IV et de Marie de Médicis », dans Les arts au temps d'Henri IV, actes du colloque, Fontainebleau, 20-21 septembre 1990, Pau 1992, pp. 331-354. M. Martin, Les Monuments équestres de Louis XIV, une grande entreprise de propagande monarchique, Paris, 1986. F. de La Moureyre, « Gobert et la statue équestre de Louis XIV dans les jardins de Ruel», Bulletin de la Société historique de Rueil-Malmaison, ${ }^{\circ} 12$, déc. 1987, pp. 21-26. Pour les statues des places royales au XVIIe siècle, voir en particulier le catalogue de l'exposition Art ou politique? Arcs, statues et colonnes de Paris, Paris, 1999 et les articles de Th. W. Gaehtgens, «La statue de Louis XIV et son programme iconographique », et d'H.
} 
pouvoir a été décrypté dans le décor sculpté, comme celui de la façade Henri II au Louvre, ou dans la statuaire du dôme central des Tuileries ${ }^{46}$. Il s'est également exprimé dans le décor de l'architecture navale, sujet de recherche assez neuf, qui a fait l'objet depuis peu d'études approfondies $^{47}$.

Le second thème de prédilection, la sculpture funéraire, est partiellement lié au premier. Ainsi, les tombeaux royaux du XVIe siècle de l'abbaye de Saint-Denis ont donné lieu à un ouvrage, à des articles, à des communications à des colloques, à des catalogues d'expositions ; pour autant, ces travaux n'répondu à toutes les questions, comme par exemple celle de l'identification et de l'emplacement des statues destinées à «la rotonde des Valois $»^{48}$. Pour le reste, les publications sont essentiellement ponctuelles, liées à un ou plusieurs monuments sculptés par un artiste ${ }^{49}$. H. Zerner a toutefois proposé une synthèse de la sculpture funéraire à la Renaissance, qui va de l'analyse problématisée des monuments et des sculptures, à leur mise en perspective dans la production artistique française au XVIe siècle $^{50}$. Pour la période suivante, on pourra se reporter à une thèse sur les monuments funéraires à Paris, qui propose une réflexion élargie à l'appréhension de la mort et à l'économie du salut en France au XVIIe siècle ${ }^{51}$.

Plus spécifiquement, c'est dans son rapport, ou plus exactement grâce à son rapport avec l'architecture que la sculpture décorative a été étudiée. La recherche s'est concentrée sur

\footnotetext{
Ziegler, «Le demi-dieu des païens. La critique contemporaine de la statue pédestre de Louis XIV », dans l'ouvrage collectif Place des Victoires, Paris, 2003, pp. 9-35 et pp. 49-65.

${ }^{46}$ V. Hoffmann, «Le Louvre de Henri II : un palais impérial», BSHAF, 1982, (1985), pp. 10-11 ; G. Bresc, «Iconologie de l'Etat monarchique : la statuaire du dôme central des Tuileries (1666-1668)», La Revue du Louvre, 1987, 1, pp. 31-38.

${ }^{47}$ Les Génies de la mer, Chefs-d'œuvre de la sculpture navale au musée national de la Marine, catalogue d'exposition, Paris, 2001 ; M. Théron, L'ornementation sculptée et peinte des vaisseaux du roi (1660-1792) (thèse de doctorat, Paris IV, 2003).

${ }^{48} \mathrm{G}$. Bresc, « Tombeaux factices de l'abbatiale de Saint-Denis ou l'art d'accommoder les restes », Bulletin de la Société nationale des Antiquaires de France, 1980-1981, pp. 114-127 ; voir les communications de Th. Lersch, A. Radcliffe et J. Thirion dans les actes du colloque Germain Pilon... op. cit.; Ph. Sénéchal, « Jean Second à Saint-Denis : les tombeaux de Charles VIII et de Louis XII en $1532 »$, Revue de l'art, 1993, 99, pp. 74-79 ; M. L. Levkoff, « Remarques sur les tombeaux de François Ier et de Henri II », dans les actes du colloque Henri II et les arts, op. cit. pp. 53-64 ; M. B. Bassett, The Funerary patronage of Catherine de' Medici : The Tomb of Henri II, Heart Monuments and the Valois Chapel, Columbia University, 1999 ; M. Beaulieu, «Pierre Bontemps et le tombeau de François Ier », Etudes d'histoire de l'art offertes à Jacques Thirion, dir A. Erlande-Brandenburg, 2001, pp. 149-162 ; les notices de G. Bresc-Bautier dans le catalogue de l'exposition Primatice,... op. cit.; les essais de G. Bresc-Bautier et Chr. L. Frommel dans Primaticcio architetto, Milan, 2005. Signalons encore la réédition en 1996 du catalogue d'exposition Le Roi, la sculpture et la mort. Gisants et tombeaux de la basilique de Saint-Denis, Archives de la Seine-Saint-Denis, 1975.

${ }^{49}$ On peut citer plus particulièrement M. Beaulieu, «La sculpture funéraire dans l'œuvre de Robert Le Lorrain (1666-1743) », dans les Actes du 106 congrès national des Sociétés Savantes (Archéologie et histoire de l'art), Paris, 1984, pp. 351-358; H. Zerner, «Germain Pilon et la tradition de la sculpture funéraire française », dans les actes du colloque Germain Pilon...op. cit., pp. 193-212 et F. de La Moureyre, «François Anguier (1604 1669) : "l'idea del bello" », GBA, 2002, 139, 1604, pp. 93-124.

${ }^{50} \mathrm{H}$. Zerner, L'art de la Renaissance en France. op. cit.

${ }^{51} \mathrm{C}$. Mazel, Les monuments funéraires à Paris (1610-1715), (Paris X-Nanterre, 2003).
} 
les quatre premières décennies du XVIe siècle ${ }^{52}$ avec des efforts remarquables pour mener des enquêtes thématiques et dégager les «systèmes » ornementaux propres à l'époque ${ }^{53}$. L'étude de l'ornement sculpté des châteaux reste toutefois en retard par rapport à celle des bâtiments mêmes ${ }^{54}$. Dans ce champ, le recours à l'informatique va certainement changer les choses, si l'on en juge par la base de données et d'images, réalisée par le centre Versailles décor sculpté extérieur sous la direction de B. Saule, qui fournit une documentation exhaustive de l'ensemble du décor sculpté extérieur des bâtiments du domaine national du château de Versailles et de Trianon.

Plus encore que la sculpture décorative, la sculpture de jardin a été étudiée de manière inégale selon les périodes. Comme on pouvait s'y attendre, pour le XVIe siècle et la première moitié du XVIIe siècle, la petite partie des sources encore dépouillée et la quasi disparition des œuvres expliquent le très faible nombre de publications ${ }^{55}$. Il en va bien autrement du règne de Louis XIV. Les jardins de Versailles et de Marly ${ }^{56}$ ont focalisé l'attention depuis

\footnotetext{
${ }^{52}$ On ne peut donner ici une bibliographie exhaustive. Le lecteur pourra néanmoins se reporter à deux colloques publiés en 2003, qui présentent plusieurs études sur le sujet: L'architecture de la Renaissance en Normandie, actes du colloque de Cerisy-la-Salle, 30 septembre-4 octobre 1998, dir. B. Beck, P. Bouet, C. Étienne et I. Lettéron, Caen, 2003 (voir en particulier J. Guillaume, «Le Candélabre en Normandie : les métamorphoses d'un ornement de 1500 à 1540 », pp. 83-98, Y. Bottineau Fuchs, "L'Ornementation "à l'antique" en Normandie au début du XVIe siècle», pp. 99-122) et Du gothique à la Renaissance..., op. cit. (voir en particulier: M. Vasselin, «Les têtes en médaillon dans le décor architectural : l'acclimatation du motif dans le Sud-Est de la France au XVIe siècle», pp. 259-270 et D. Leloup, «L'introduction des formules de la Renaissance avant les années 1540 en Bretagne », pp. 163-177. Ajoutons encore B. Tollon, «Le château d'Assier » et «L'église d'Assier », Congrès archéologique de France, 1989, v. 147, pp. 137-149, et pp. 125-136, et I. Lettéron, D. et Gillot, Rouen. L'hôtel de Bourgtheroulde. Demeure des Le Roux. Cahiers du patrimoine, nº44, Paris, 1996 ; C. Chédeau, Les Arts à Dijon ... op. cit, pp. 160-169.

${ }^{53} \mathrm{~J}$. Guillaume a dirigé plusieurs travaux d'étudiants sur le décor sculpté à la Renaissance (nous mentionnons ici ceux qui n'ont pas encore donné lieu à une publication) : V. Drocourt, Le Candélabre en Italie du Nord et en Normandie (Paris IV, 1998) ; É. Dagnas-Thomas, Le système ornemental de la première Renaissance française (Thèse, Tours, CESR, 1998) et C. Titeux, Le mur orné : bossages, tables, bas-reliefs et autres enrichissements, dans l'architecture française de 1540 à 1770 (thèse, Paris IV, en cours). Pour le XVIIe siècle, les études semblent moins nombreuses : P.-A. Lamy, Utilisation des ordres et ornements dans la composition des façades aux XVIIe et XVIIIe siècles : principes et détournements (DEA, Paris VIII, 2000).

${ }^{54}$ A noter l'article exemplaire de C. Mouchebœuf-Guiorgadzé, «Les chapiteaux de Chambord : recherches sur la stylistique ornementale de la premier renaissance », Revue de l'art, 1999, n 124, pp. 33-42, A. Cojannot, « Le Bas-relief à l'antique dans l'architecture parisienne du XVIIe siècle : du Louvre de François Sublet de Noyers à celui de Jean Baptiste Colbert », Studiolo : revue d'histoire de l'art de l'Académie de France à Rome, 2002, 1, pp. 20-40. Mentionnons aussi le Mémoire de l'Ecole du Louvre de B. Garnier, «Le décor sculpté du château de Vaux-le-Vicomte de 1656 à nos jours (1989).

${ }^{55} \mathrm{G}$. Bresc, «Fontaines laïques de la première Renaissance française : les marbres de Tours, de Blois et de Gaillon », dans Sources et fontaines du Moyen Âge à l'Âge baroque, actes du colloque tenu à l'Université Paul Valéry (Montpellier III), les 28-30 novembre 1996, Paris, 1998 ; F. Bardati, «La Grotte des Pins à Fontainebleau », dans Artefici d'acque e giardini. La cultura delle grotte e dei ninfei in Italia e in Europa. Atti del V convegno internationale sui parchi e i giardini storici, Florence et Lucques, 1998, éd. Florence 1999, pp. 39-47.

${ }^{56}$ B. Rosasco, The Sculptures of the Château de Marly during the reign of Louis XIV, Ph. D., New-York University, 1980, University Microfilms International, Ann Arbor (Mich.), 1982 ; id., « The sculpture of Marly and the Programme of Versailles », Journal of Garden History, 1983, pp. 301-316. Catalogue d'exposition Les Chevaux de Marly, Marly-le-Roi Louveciennes, Musée-Promenade, 19 octobre-15 décembre 1985.
} 
plusieurs années. Des chercheurs étrangers ont, dans le sillage de P. Francastel, distingué les grandes étapes du développement de la statuaire ${ }^{57}$, mis en évidence le rôle joué par le peintre ou l'architecte, approfondi l'étude iconographique, et révélé les rapports des sculptures aux bâtiments et aux jardins ${ }^{58}$.

En fin de compte, ce sont peut-être les relations entre la sculpture et la peinture qui, contre toute attente, sont les moins traitées. Certes, une place est parfois réservée à la sculpture dans des expositions de peinture (elle n'est pourtant pas convoquée sur des sujets où on l'attendrait comme le montre ici G. Bresc-Bautier), mais elle est souvent restreinte et l'analyse des liens et des échanges entre les deux arts, quasiment absente des catalogues. Le sujet a, semble-t-il, été abordé plus fréquemment à partir des textes que des œuvres, bien que les premiers soient peu prolixes sur la sculpture ${ }^{59}$. La notion de paragone, si souvent évoquée, n'aurait-elle pas limité plus que stimulé la réflexion?

L'étude des rapports de la sculpture française des XVIe et XVIIe siècles avec les autres formes artistiques est, plus qu'ailleurs, un moyen pour mieux comprendre les œuvres. Pour un art dont l'invention est, par tradition, généralement définie par un autre artiste que le sculpteur même, un peintre ou un architecte, pour un art qui, par nature, est plus étroitement dépendant de la statuaire antique et, pour la période étudiée, de l'œuvre sculpté de Michel-Ange et du Bernin, mais dont l'accès à ces modèles s'est souvent fait par l'intermédiaire de reproductions, pour un art enfin qui a pu bénéficier de la diffusion de l'estampe, on attend des recherches sur l'identification, la circulation et la réception des modèles, sur les échanges artistiques, avec l'Italie et la Flandre en particulier, enfin sur l'évolution des formes ainsi engendrées. Des travaux de différentes natures, de l'étude ponctuelle à la synthèse, vont dans

\footnotetext{
${ }^{57}$ A. Friedman, The 'Grande Commande' for the Sculpture of the Parterre d'eau at Versailles, 1672-1683, Minneapolis, 1983, id.« The evolution of the Parterre d'eau », Journal of Garden History, 1988, pp. 1-30 ; J. Girard, Versailles Gardens. Sculpture and Mythology, Londres, 1985 ; Th. Hedin, « The Petite commande of 1664 : burlesque in the gardens of Versailles », The Art bulletin, 2001, 83, 4, pp. 651-685 (version française dans Versalia, 2004, 7, pp. 74-111).

${ }^{58}$ G. Weber, Brunnen und Wasserkünste in Frankreich im Zeitalter von Louis XIV, Worms, 1985 ; Th. Hedin, «Le Nostre to Mansart. Transition in the Gardens of Versailles », GBA, 1997, pp. 191-344 ; J.-Cl. Boyer, « La Statuaire des jardins de Versailles : Mignard maître d'œuvre», Versalia, 1999, 2, pp. 46-59 ; P. Schneider, «Die komposite Welt des Parterre d'Eau der Gartenanlage von Versailles 1672-1683. Charles Le Brun im Spannungsfeld von Kunst und Wissenschaft », Die Gartenkunst, 2000, pp. 257-274. Un colloque sur la sculpture versaillaise (Versailles, mai 2004) devrait être publié prochainement dans Versalia.

${ }^{59}$ Pour le XVIIe siècle, on peut signaler l'article d'A. Magnien, « Des effets et des causes. Imiter la nature dans la sculpture du XVIIe siècle », dans les actes du colloque, The Muse in the marble : Plastic Arts and Aesthetic Theories in the Seventeenth Century (Rome, février 2004) à paraître en 2005 aux University of Delaware Press. Le mémoire de maîtrise d'E. Delapierre, De la théorie esthétique à l'œuvre d'art : l'exemple de Michel Anguier, sculpteur et académicien (Paris X-Nanterre, 1995) n'est pas publié. Certains ouvrages traitent pour partie de la sculpture française du XVIIe siècle, ainsi J. Lichtenstein, La tache aveugle, Essai sur les relations de la peinture et de la sculpture à l'âge moderne, Paris, 2003 et A. Magnien, La nature et l'antique, la chair et le contour, essai sur la sculpture française au XVIIIe siècle, Oxford, 2004.
} 
ce sens. L'article pionnier de S. Pressouyre sur les fontes de Primatice a suscité des réflexions sur les rapports de la sculpture française du XVIe siècle à l'antique ${ }^{60}$. On retrouve ce thème au XVIIe siècle, dans les années 1640-1660 avec les campagnes de moulage à Rome et l'enthousiasme éphémère pour les bas-reliefs à l'antique ${ }^{61}$, et quelque temps après dans la statuaire de Versailles. Plus largement, il soulève des débats sur le choix du modèle privilégié, la nature ou l'antique, derrières lesquels se profilent des oppositions entre une conception italienne et une conception française, entre Anciens et Modernes, et au-delà entre Paris et Rome $^{62}$. En dehors des chantiers royaux et de Paris, certains centres provinciaux ouverts aux échanges internationaux constituent des terrains féconds pour l'étude de la circulation et de la transcription de modèles étrangers en particulier dans la sculpture, comme cela vient d'être démontré pour la Champagne méridionale à la fin du XVe siècle et au début du XVIe siècle ${ }^{63}$. Dès la Renaissance, l'estampe fut incontestablement l'un des premiers vecteurs de diffusion des modèles. Sa réception varie néanmoins selon le type de sculptures concernées : que les reliefs sur bois montrent une prédilection pour l'utilisation de modèles gravés ${ }^{64}$ s'explique sans doute en partie car cette technique, surtout ornementale, relève plus du savoir faire du praticien que du sculpteur.

L'interprétation de la réception du modèle formel est un exercice délicat, celle du style l'est plus encore. Les travaux sur la fortune d'un ou plusieurs sculpteurs dans le monde de

\footnotetext{
${ }^{60}$ S. Pressouyre «Les fontes de Primatice à Fontainebleau », Bulletin Monumental, 127, III, 1969, pp. 223-239. L. Chatelet-lange, «Die Statue «à l'antique» im französischen Garten des 16. Jahrhunderts : zur Renaissance eines plastischen Themas », Österreichische Zeitschrift für Kunst und Denkmalpflege, vol 41, n³-4, 1987, pp.97-105 ; F. Bardati, «Les bronzes d'après l'Antique de Fontainebleau et la sculpture française au milieu du XVIe siècle », GBA, CXXXVI, novembre 2000, pp. 159-168 et B. Jestaz, "Les premières copies d'antiques », dans D'après l'antique, Paris, musée du Louvre, 16 octobre 2000 - 15 janvier 2001, Paris, 2000, pp. 45-52.

${ }^{61}$ A. Le Pas de Sécheval, «Les mission romaines de Paul Fréart de Chantelou en 1640 et 1642 : à propos des moulages d'antiques commandés par Louis XIII », XVIIe siècle, 3, 172, 1991, pp. 259-274 ; G. Bresc "Copier l'antique à la cour de Louis XIV », dans D'après l'antique, op. cit, pp. 60-68 et id., "Ces bas-reliefs ne sont d'aucun usage dans ce pays-ci », dans L'Idéal classique. Les échanges artistiques entre Rome et Paris au temps de Bellori (1640-1700), actes du colloque, dir. O. Bonfait, Rome, Villa Médicis, 7-9 juin 2000, Paris et Rome, 2002, pp. 299-316 ; A. Cojannot, op. cit.

${ }^{62}$ Sur ces questions, on se reportera en particulier à Chr. Michel, « Le chevalier Bernin et l'enseignement artistique en France ", dans Le Bernin et l'Europe. Du baroque triomphant à l'âge romantique, acte du colloque international, Paris, 1998, dir. Ch. Grell et M. Stanic,, Paris, 2002, pp. 95-105 ; J. Lichtenstein, La tache aveugle, op. cit..., chap. I.

${ }^{63} \mathrm{~V}$. Boucherat, Recherches sur le rôle des modèles dans la production artistique en Champagne méridionale à la fin du Moyen Âge (v. 1485-v. 1535), (thèse de doctorat, Paris IV, 2001, en cours de publication). Ce travail a inspiré l'identification de sources dans un autre foyer en relation étroite avec l'Italie, voir Chr. Gallissot-Ortuno, «Aux sources de la Renaissance provençale: étude des sibylles de la cathédrale Saint-Sauveur d'Aix-enProvence », dans Du gothique à la Renaissance..., op. cit., pp. 33-50.

${ }^{64}$ J. Thirion, « Le credo du musée d'Écouen. Panneaux sculptés d'après Martin de Vos », Revue de l'art, $\mathrm{n}^{\circ} 40$ 41, 1978, pp. 109-120 ; G. Bresc-Bautier, «Le musée imaginaire de Pierre Vaneau, sculpteur du Puy (16531694) », Revue de l'art, 1990, n 87, pp. 59-83. Citons également le mémoire de recherche de l'Ecole du Louvre de J. Langlois, Les Stalles de Saint Vaast d'Arras : contribution à l'histoire de la sculpture sur bois et de ses sources gravées (sous la dir. de J.-R, Gaborit, 1994).
} 
l'art, et inversement de la réception d'un ou plusieurs artistes dans la sculpture sont encore rares $^{65}$ et concernent d'abord des figures majeures comme Bernin ${ }^{66}$.

Il peut paraître plus laborieux à priori de travailler sur la sculpture que sur les autres arts. Elle offre moins d'œuvres et moins de textes à l'historien. Difficile à photographier, elle est mal reproduite. Pour autant, les travaux récents se montrent capables de renouveler des sujets traditionnels, mais également d'élaborer de nouvelles problématiques. Plus largement, ils invitent à penser différemment la sculpture, en ouvrant parfois des limites chronologiques non adaptées, en affinant la réflexion sur l'œuvre par un travail sur sa forme et sur sa matière, ou en abordant par plusieurs voies les rapports qu'elle entretient avec les autres arts. Il serait temps de dépasser une hiérarchie des arts qui a singulièrement bridé la recherche, d'arrêter de se plaindre du manque d'études sur la sculpture et de s'y atteler !

\footnotetext{
${ }^{65}$ E. Passignat conduit actuellement une thèse sur La réception de la sculpture maniériste en France au XVIIe et XVIIIe siècle (Strasbourg II) (précisons qu'il s'agit de la sculpture réalisée en France au XVIe siècle par des sculpteurs tant français qu'italiens).

${ }^{66}$ Voir en particulier les interventions de P. Julien, «Edifiantes souffrance : l'agonie extatique, du Bernin à Pierre Legros», F. Cousinié, «De la morbidezza du Bernin au «sentiment de la chair» dans la sculpture française », K. Herding, Pierre Puget : le Bernin de la France ou subtil antiberninien ? », et M. Boudon, «L'antithèse Bernin-Du Quesnoy dans la littérature artistique française », dans Le Bernin et l'Europe... op. cit, pp. 259-349. La réédition annotée du Journal de voyage du cavalier Bernin en France de Chantelou par M. Stanic (Paris, 2001) a contribué en outre à la connaissance du séjour de l’Italien en France.
} 